\title{
Lack of consistent individual differences in rats on tasks that require response inhibition
}

\author{
PHIL REED and LISA PIZZIMENTI \\ University College London, London, England
}

\begin{abstract}
Hungry rats performed four different tasks which all required responding to be withheld for food reinforcement to be obtained. The tasks were the following: an omission schedule, a go/no-go procedure, a differential reinforcement of low rate schedule, and a choice task in which the subject could respond for a small immediate reward or a large delayed reward. Each rat experienced each task twice. For each task, the rank ordering of the subjects' performance on the test and retest was highly consistent, indicating the reliability of the task for measuring a particular "ability." However, there were no consistencies in the performance of individual rats between tasks. This suggests that claims regarding consistent individual differences in brain-lesioned nonhumans across a range of such tasks are not supported in intact subjects.
\end{abstract}

Approaches to analyzing personality may be divided into two broad categories. One approach suggests that individuals may possess one out of a number of personality traits that are stable across different situations (e.g., Eysenck \& Eysenck, 1985; Gray, 1987). This might be termed a trait approach. In contrast, the state approach suggests that behavior is more consistent in the same situation than across different situations (see Mischel, 1990; Shoda, Mischel, \& Wright, 1993). Nonhumans as well as humans have been used to explore the mechanisms that might underlie personality (e.g., Mather \& Anderson, 1993; Wanchisen, Tatham, \& Mooney, 1989). In fact, some models of personality in humans draw extensively on work involving nonhuman performance in conditioning tasks (see, e.g., Gray, 1987). However, little work has been done to establish whether data collected from nonhuman subjects may be best characterized as supporting the state or trait view of personality.

An example of a model of personality based on data from studies with nonhumans (especially rats) is that of Gray (1987). These data suggested a three-factor model of personality, involving an activation system that deals with behavior elicited by rewards; an inhibition system that deals with responses to punishment and the inhibition of ongoing behavior; and a flight/fight system which mediates the behavioral effects of unconditioned aversive events. Since damage to the septum and to the hippocampus has been found to produce impairments on a variety of tasks (see Gray, Rawlins, \& Feldon, 1979, for a review), it

Part of this work formed a thesis submitted in partial fulfillment of a Master of Science degree for the second author. Thanks are due Celia Heyes and Lisa Osborne and two anonymous reviewers for comments on an earlier version of this paper. Requests for reprints should be sent to: P. Reed, Department of Psychology, University College London, Gower Street, London WC1E 6BT, England (e-mail:ucjtspr@ucl.ac.uk).

-Accepted by previous editor, Vincent M. LoLordo has been assumed that performance on those tasks was mediated by this common substrate, and that this may underlie the expression of a personality trait. If this point is accepted, it might provide support for a trait view of personality (see, e.g., Gray, 1987).

However, it is possible that the tasks employed to assess nonhuman performance have not, in fact, relied on the operation of a single underlying system. The ability to perform each of the tasks may have been independent of the abilities to perform the others, so that these abilities share nothing more than a similarity of description on the part of the experimenter. In principle, a rather gross level of physiological intervention could disrupt a relatively general process, which, in turn, could affect performance on a series of tasks that are primarily based on separate abilities. If this were the case, and performance on these tasks is largely independent, this would provide the basis for evidence in support of a state view of personality.

To better assess these two alternative views of personality based on results with nonhuman subjects, use of techniques employed in the investigation of human individual differences might be employed. Such investigations often proceed with the aid of factor analysis that isolates commonalities in the behavior observed in subjects under a variety of conditions (see Cattell, 1965). Such an approach is not without precedent in the literature concerning individual differences in nonhumans (see Huntingford, 1976; Mather \& Anderson, 1993). This analysis would identify whether the performance on a number of tasks shares common features, indicated by the existence of only a few factors contributed to by all tasks, or whether the performance on the tasks is independent, indicated by the existence of factors contributed to by only one of the tasks.

The present study focused on whether tasks typically used to study inhibitory systems in conditioning procedures could offer any evidence for the existence of stable behavioral traits in nonhumans. This particular dimension was chosen for examination on the basis of the results of 
a study reported by Wilson, Barrett, and Gray (1989). Wilson et al. (1989) attempted to establish stable traits in human subjects, which were putatively equivalent to those established in the investigation of nonhumans (e.g., approach, avoidance, flight/fight). This study found little evidence in support of Gray's (1987) three-factor model. The only behaviors which appeared to be correlated to any extent in the human case in the report by Wilson et al. (1989) were those concerned with the putative inhibition system. Given this, it seems appropriate to use tasks requiring responding to be inhibited in order to further investigate whether in intact nonhuman subjects there are correlations between performance on a variety of tasks predicted by Gray's (1987) model.

One suggestion made to explain the lack of equivalence between human and nonhuman subjects by Wilson et al. (1989) was that the tests with the human subjects did not map onto the behavior exhibited by nonhumans on which the personality model was based. Of course, a further important difference between the nonhumans used in previous reports and the human subjects studied by Wilson et al. (1989) is that the humans did not have brain damage comparable to that of the nonhumans. It could be that in the absence of a physiological intervention, there are no consistent individual differences along the dimensions suggested by Gray (1987).

The present study, therefore, had two objectives: first, to determine whether there were any common factors implicated in the performance of rats across a variety of tasks that had a certain face validity in assessing inhibition of ongoing responses (and by implication the inhibition system in Gray's, 1987, model); and second to see whether rats' performance could be said to reflect any individual differences that were consistent across a variety of tasks, or whether performance was task specific and, hence, more supportive of a state approach to personality.

\section{METHOD}

\section{Subjects}

Thirty-two experimentally naive, male Lister hooded rats were used in the present experiment. The rats were 3-4 months old at the start of the study, had a free-feeding body weight range of $310-395 \mathrm{~g}$, and were maintained at $85 \%$ of this weight throughout the experiment. The animals were housed in groups of 4 , with water constantly available in the home cage.

\section{Apparatus}

Four identical operant conditioning chambers (Campden Instruments) were used. Each chamber was housed in a light- and soundattenuating case and was ventilated by a fan that provided background masking noise $(65 \mathrm{~dB}[\mathrm{~A}])$. Each chamber had two levers, both of which were permanently inserted into the chamber. Each lever was made of transparent Perspex and had a light bulb mounted behind it, outside the chamber, which could be used to illuminate the lever. Reinforcement (which consisted of one $45-\mathrm{mg}$ food pellet) was delivered to a centrally located, recessed, food tray that was covered by a clear Perspex, hinged flap. The magazine tray could be illuminated from behind by a small bulb. A jewelled houselight was located on the ceiling of the chamber and another was located in the center of the wall containing the magazine tray and levers. Both lights could be used to provide a visual stimulus

\section{Procedure}

Subjects were magazine trained in two 30 -min sessions, during which food was delivered according to a variable time $60-\mathrm{sec}$ schedule. For the first session, the magazine flap was taped open to allow easy access to the food pellets. During the second session, and for all future sessions, the flap was lowered to its standard resting position. Following magazine training, subjects were taught to press the lever in one session, during which every response to each of the two levers was reinforced (i.e., a concurrent continuous reinforcement, or CRF, schedule was in operation). This and all subsequent sessions lasted for $30 \mathrm{~min}$. Each of the eight phases of the experiment lasted for 20 sessions.

Omission schedule. For the first and fifth phases of the study, the subjects were exposed to an omission schedule. During this phase, a trial consisted of the illumination of the magazine tray for $10 \mathrm{sec}$. Following this stimulus presentation, reinforcement was delivered if the subject had not entered the magazine during the stimulus presentation. If a subject had made a tray entry response, no food was delivered following termination of the stimulus. The intertrial interval (ITI) between tray light illuminations was $80 \mathrm{sec}$. There were 20 stimulus presentations during a session

Go/no-go training. Immediately following exposure to the omission schedule (i.e., during Phases 2 and 6 of the experiment), subjects were exposed to a go/no-go schedule. During these phases of the study, subjects were exposed to two visual stimuli: one was the jewelled light located on the wall of the chamber above the magazine tray, which served as the go stinulus; the other was the overhead light, which served as the no-go stimulus. Each stimulus was presented 10 times during the session, and the two were randomly ordered. During a go trial, the stimulus was illuminated for $10 \mathrm{sec}$, and if the subject made a tray entry response during this time, reinforcement was delivered after stimulus termination. For no-go trials, the other stimulus was presented for $10 \mathrm{sec}$. Reinforcement was presented following the termination of the stimulus only if the subject had not made a tray entry response during the stimulus presentation. If the subject made a response during the no-go stimulus presentation, no food was delivered at stimulus offset. The ITI during these sessions was $80 \mathrm{sec}$

Differential reinforcement of low-rate training. During Phases 3 and 7 of the experiment (immediately following go/no-go training), the left lever was illuminated, and subjects were exposed to a differential reinforcement of low rate (DRL) 20-sec schedule. The temporal requirement of this schedule was introduced immediately: there was no gradual increase in the temporal parameter of the schedule. During the DRL session, subjects received reinforcement only if their responses were separated by a minimum of $20 \mathrm{sec}$, or if a response was made a minimum of $20 \mathrm{sec}$ after a reinforcer had been delivered. Any responses emitted which did not satisfy one of these criteria reset the time requirement.

Choice training. During Phases 4 and 8 (which immediately followed DRL training), subjects were exposed to a two-lever choice task. Each lever was associated with one component of a multiple fixed ratio (FR) 5, FR-5 schedule. The operation of a particular component of the multiple schedule was signaled by the illumination of a response lever. Each lever was always associated with a particular component during training. One of the components led to the delivery of a small immediate reinforcer. The other component led to the delivery of a large delayed reinforcer. At the start of a session, one of the levers was illuminated; this lever was randomly determined at the beginning of each session. The subject completed the FR-5 requirement, and the lever was darkened. If the component was associated with the small immediate reward, one food pellet would be delivered immediately on completion of the response requirement. The levers remained darkened for $15 \mathrm{sec}$. If the component was that associated with the large delayed reward, the lever was darkened, and after $5 \mathrm{sec}$, four food pellets were delivered. The levers remained darkened for a further $10 \mathrm{sec}$. After the period in which the levers were darkened, the lever not illuminated initially 
was illuminated, and the subject completed the FR-5 requirement This lever was then darkened, the appropriate reward was delivered, and the ITI commenced. The lever initially illuminated was then illuminated again, the subject completed the component requirement, and the appropriate reward was delivered. The lever illuminated second in the session was then illuminated again, and the same procedure was followed. Following the completion of four components (two of each type), both levers were illuminated and the subject was presented with a choice between the components. The first response to either lever darkened the lever that was not chosen (i.e., that not responded to), and the subject had to complete the schedule requirement on the chosen lever. Following completion of the response requirement, the reinforcement appropriate for that condition was delivered. After this choice probe trial, the lever not chosen in the choice test was illuminated, and the subject was required to complete this schedule component. This ensured equal amounts of exposure to the two types of component. This training constituted a block. There were six such blocks of training during a session.

\section{RESULTS}

The results are based on the subjects' mean performance over the last three sessions of each phase of the study. It was hoped that in the last three sessions, performance may have become relatively stable, while the influence of performance generated by the previous contingency may have been minimized.

\section{Response Rates on Each Contingency}

Omission. On the first exposure to the omission contingency, subjects made a mean 35.65 tray entry responses during the stimulus presentation, with a standard error of the mean (SEM) of 3.04. Individual scores varied between 12.67 and 97.33 mean responses per session. The distribution of scores was positively skewed (skewness $=2.14$ ).

On the second exposure to the omission contingency, subjects responded a mean 50.78 times per session, with an $S E M$ of 3.48. Mean individual scores varied between 20.33 and 111.67 responses per session. The distribution of scores was slightly positively skewed (skewness $=1.48$ ).

To test the reliability of the omission task, a Spearman's correlation coefficient was calculated for the subjects' performance on the two exposures to the contingency. This revealed a significant correlation between performance on the two exposures $(\rho=.897, p<.001)$.

Go/no-go. Only data from the no-go trials were considered for the present purposes. On the first exposure to this contingency, subjects responded a mean $7.59 \pm 0.84$ times during no-go trials. Individual mean scores varied from 0 to 30.67 responses per session during the no-go trials. The distribution of scores was positively skewed (skewness $=3.13$ ).

On the second exposure to the contingency, subjects responded a mean $10.60 \pm 0.91$ times per session during the no-go trials. Individual scores varied between a mean of 0 and 23.33 responses per session. There was little skewness in the distribution (skewness $=0.13$ ).

To test the reliability of the no-go task, a Spearman's correlation coefficient was calculated for the subjects' mean performance on the two exposures to the contin- gency. This revealed a significant correlation between performance on the two exposures $(\rho=.368, p<.05)$.

DRL. On the first exposure to the DRL schedule, subjects responded a mean $118.18 \pm 10.41$ times per session. Individual mean scores varied from 32.33 to 227.33 responses per session. There was little skewness in the distribution (skewness $=0.57$ ).

On the second exposure to the schedule, subjects responded a mean $149.28 \pm 10.48$ times per session. Mean individual scores varied between 62.67 and 306.33 responses per session. There was little skewness in the distribution (skewness $=0.816$ ).

To test the reliability of the DRL schedule, a Spearman's correlation coefficient was calculated for the subjects' mean performance on the two exposures to the contingency. This revealed a significant correlation between performance on exposures $(\rho=.869, p<.001)$.

Choice training. Only the number of times that the smaller immediate reward was chosen on the choice trials was considered for the present purposes. On the first exposure to the "self-control" procedure, subjects chose the smaller immediate reward a mean $3.96 \pm 0.21$ times per session. Individual mean scores varied from 0 to $5.67 \mathrm{re-}$ sponses per session. There was little skewness in the distribution (skewness $=-0.89$ ).

On the second exposure to the schedule, subjects responded a mean $3.84 \pm 0.24$ times per session. Mean individual scores varied between 0 and 5.33 responses per session. There was little skewness in the distribution (skewness $=-0.408$ ).

To test the reliability of the self-control measure, a Spearman's correlation coefficient was calculated for the subjects' mean performance on the two exposures to the contingency. This revealed a significant correlation between performance on the two exposures $(\rho=.766, p<$ $.001)$.

\section{Normalization}

Since the different tasks used different measures to assess performance, the data were subject to a normalization function. This procedure also had the advantage of restoring normality to a number of the test distributions which appeared to be markedly skewed. In fact, Shapiro-Wilks tests conducted on all eight phases revealed that both omission tests and the first exposure to the go/no-go task produced distributions that deviated significantly from normal [highest Shapiro-Wilks $(32)=0.86$, all $p \mathrm{~s}<.10$ ] A Blom normalization function, $(r-3 / 8) /(n+1 / 4)$, was applied to the mean data from the last three sessions of each of the phases of the study. After this procedure all phases were normally distributed (all Shapiro-Wilks $>0.99$ ), with a mean of 0 and an SEM of 0.172 .

\section{Factor Analysis: Common Factors in the Tasks}

The normalized data were then analyzed to ascertain whether any of the tasks correlated significantly with one another. The full correlation matrix is displayed in Table 1.

Inspection of this correlation matrix reveals that, with the exception of the correlation between the second expo- 
Table 1

Correlation Matrix for the Normalized Scores

\begin{tabular}{lcccrccc}
\hline & Om2 & Nogo1 & Nogo2 & DRL1 & DRL2 & \multicolumn{1}{c}{ SCl } & SC2 \\
\hline Om1 & $0.805 \dagger$ & 0.303 & 0.130 & -0.012 & 0.008 & 0.185 & 0.336 \\
Om2 & & 0.042 & 0.092 & 0.280 & 0.215 & 0.083 & 0.152 \\
Nogo1 & & & $0.467^{*}$ & -0.054 & 0.055 & 0.125 & 0.247 \\
Nog02 & & & & -0.044 & -0.029 & $0.435^{*}$ & 0.238 \\
DRL1 & & & & & $0.880 \dagger$ & -0.115 & -0.216 \\
DRL2 & & & & & & -0.072 & -0.070 \\
SC1 & & & & & & & $0.726 \dagger$ \\
\hline
\end{tabular}

Note-Om, omission; Nogo, go/no-go schedule; DRL, differential reinforcement of low rate; SC, self-control. For further explanation, see text. ${ }^{*} p<.01 . \dagger p<.001$.

sure to the go/no-go and the first exposure to the self-control contingency, the only significant correlations were between the first and second exposures to the same contingency. For these analyses it should be noted that a rejection criterion of $p<.01$ was used. The use of multiple correlations increases the chances of a Type I error, and the employment of a more conservative rejection criterion than the $p<.05$ adopted elsewhere in this paper goes some way to guard against this possible source of error.

To determine the existence of common factors in the performance produced by these tasks, the normalized data were subjected to a principal component factor analysis with varimax rotation. The results of these analyses are displayed in Table 2. The initial factor analysis revealed four factors with eigenvalues greater than one; together, these factors accounted for $87 \%$ of the variance. These factors were selected for rotation. Following the varimax rotation, it was found that two variables loaded onto each factor to a much greater extent than did the other variables (loadings $=0.70$; all other loadings $<0.35$ ). The two exposures to the DRL schedule loaded onto Factor 1, the two exposures to the omission contingency loaded onto Factor 2 , the two exposures to the self-control procedure loaded onto Factor 3, and the two exposures to the go/nogo procedure loaded onto Factor 4.

\section{Cluster Analysis: Individual Differences in Performance}

A cluster analysis was performed to reveal whether any sets of subjects performed consistently well or badly across the tasks. Since the results of the factor analysis revealed that there were four factors, which corresponded to the four tasks, the mean performance of the subjects on each task (first and second exposure combined) was used

Table 2

Results of Principal Components Factor Analysis

\begin{tabular}{|c|c|c|c|c|c|}
\hline \multirow[b]{2}{*}{ Variable } & \multicolumn{5}{|c|}{ Initial Statistics } \\
\hline & Communality & Factor & Eigenvalue & $\%$ Var & Cum $\%$ Var \\
\hline $\mathrm{Oml}$ & 0.943 & 1 & 2.494 & 31.2 & 31.2 \\
\hline $\mathrm{Om} 2$ & 0.912 & 2 & 2.102 & 26.3 & 57.5 \\
\hline Nogol & 0.817 & 3 & 1.335 & 16.7 & 74.1 \\
\hline Nogo2 & 0.711 & 4 & 1.061 & 13.3 & 87.4 \\
\hline DRLl & 0.950 & 5 & 0.614 & 7.7 & 95.1 \\
\hline DRL2 & 0.930 & 6 & 0.197 & 2.5 & 97.5 \\
\hline $\mathrm{SCl}$ & 0.900 & 7 & 0.116 & 1.4 & 99.0 \\
\hline SC2 & 0.827 & 8 & 0.081 & 1.0 & 100.0 \\
\hline \multicolumn{6}{|c|}{ Rotated Factor Matrix (Varimax Rotation) } \\
\hline & Factor 1 & & & Factor 3 & Factor 4 \\
\hline Om1 & -0.662 & 0 & & 0.144 & 0.170 \\
\hline Om2 & 0.214 & & & 0.060 & -0.020 \\
\hline Nogol & -0.023 & & & 0.003 & 0.892 \\
\hline Nogo2 & 0.016 & & & 0.317 & 0.781 \\
\hline DRL1 & 0.964 & & & -0.111 & -0.043 \\
\hline DRL2 & 0.962 & & & -0.025 & 0.031 \\
\hline $\mathrm{SCl}$ & -0.023 & & & 0.936 & 0.153 \\
\hline $\mathrm{SC} 2$ & -0.118 & & & 0.872 & 0.122 \\
\hline \multicolumn{6}{|c|}{ Factor Transformation Matrix } \\
\hline & Factor 1 & & & Factor 3 & Factor 4 \\
\hline Factor 1 & -0.114 & & & 0.673 & 0.501 \\
\hline Factor 2 & 0.867 & & & -0.192 & -0.029 \\
\hline Factor 3 & 0.457 & & & 0.303 & 0.445 \\
\hline Factor 4 & -0.163 & & & -0.646 & 0.741 \\
\hline
\end{tabular}

Note-Om, omission; Nogo, go/no-go schedule; DRL, differential reinforcement of low rate; $\mathrm{SC}$, self-control. For further explanation, see text. 


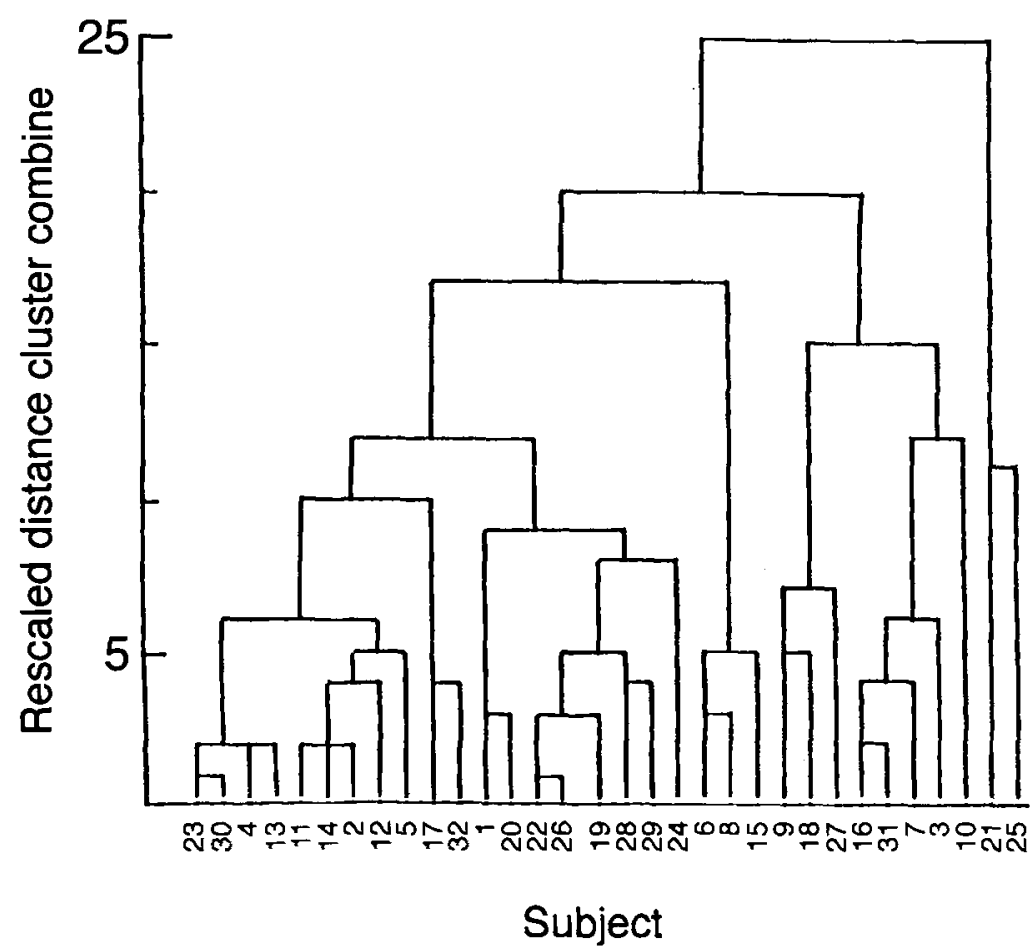

Figure 1. A dendrogram produced by the eluster analysis, based on an agglomeration schedule using the average linkage (between groups).

for the cluster analysis. The factor loadings were not employed, since it is not clear from the present analysis whether these loadings were stable, but it was clear that each factor had only two contributing variables. An agglomeration schedule using the average linkage (between groups) produced the dendrogram of the cluster analysis displayed in Figure 1.

This method of data analysis (see West, 1991, pp. 153158 ) displays the results in such a way that, for example, Subject 23 can be seen to be most like Subject 30, and, taken as a unit, these 2 subjects can be seen to be most like the unit comprising Subjects 4 and 13. That is, the subjects (and units of subjects) are considered to be like each other, if their scores across the tasks display close similarity to one another. Inspection of these results suggests that there were two relatively coherent clusters of subjects and a number of subjects that exhibited no affinity to any particular cluster. The first cluster comprised Subject 23 down to Subject 32; the second Subject 1 down to 24 . The subjects below this in the dendrogram fell into several rather disjointed clusters. In order to characterize the clusters, the mean scores of the subjects in each cluster were calculated. Subjects were assigned to clusters by using the clusters with the smallest average linkage. Seven clusters were chosen after experimenting with different numbers of clusters, since this was the smallest number which allowed differentiation of the two main clusters (Subjects 23-32, and Subjects 1-24). The mean, normalized scores for each of these clusters on the four factors (tasks) are shown in Table 3.
From these data, it is apparent that no one cluster performed uniformly well or badly (i.e., no cluster has means which are all positive or all negative) across these tasks. Rather, the clusters were formed of subjects that performed well on a particular task and poorly on other tasks.

\section{DISCUSSION}

The present results are clear in their implications for the different views of individual differences. The trait view suggests the existence of consistent patterns of performance by subjects across tasks. The results from the factor analysis revealed that performance on the four tasks used in this study showed little in common. If a stable trait was responsible for individual differences in performance, the factor analysis would be expected to reveal a single factor, onto which all, or many, of the tasks loaded. In contrast, the fact that the four different tasks loaded very pre-

Table 3

Mean Scores on Each Factor (Task) for the Seven Clusters

\begin{tabular}{ccrcrr}
\hline Cluster & Subjects & Om & Nogo & DRL & \multicolumn{1}{c}{ SC } \\
\hline 1 & $1-24$ & -0.71 & 0.093 & -2.60 & 0.32 \\
2 & $23-32$ & -0.68 & -1.13 & 0.62 & -0.60 \\
3 & $16-10$ & 0.66 & 0.51 & -0.74 & 0.39 \\
4 & $6-15$ & -1.20 & 1.73 & 1.92 & -0.72 \\
5 & $9-27$ & -2.07 & 1.13 & 3.41 & 0.55 \\
6 & 10 & 0.42 & -0.87 & 1.71 & 0.09 \\
7 & $21-25$ & 3.71 & 1.08 & 2.41 & -1.26 \\
\hline
\end{tabular}

Note-Om, omission; Nogo, go/no-go schedule; DRL, differential reinforcement of low rate; SC, self-control. 
cisely onto four different factors suggests that performance on one task was not related to performance on the other tasks.

Although the present tasks had little in common with each other in terms of the subjects' performance, it could still be claimed that some subjects were good at all these tasks and that some subjects were bad at these tasks, perhaps because of differing capabilities of their separate response inhibition systems. Yet the results of the cluster analysis revealed that none of the clusters of subjects produced in the present analysis displayed a consistent tendency to perform well or poorly across tasks. Rather, these clusters appear to be composed of subjects that performed well on some particular tests and badly on other specific tasks.

These findings thus provide no evidence for any consistent stable individual differences obtained between rat subjects on response inhibition tasks. If anything, these data support the state view of individual differences, which claims that performance is determined by the situational demands rather than any enduring trait; the individual differences in performance on a particular task were relatively stable in the present study. The implications for these findings in terms of the physiological systems that may underlie such performance will be addressed after discussion of the reliability and validity of the tasks used in this experiment.

The separate analyses of the performance on the four different tasks revealed that the rank ordering of subjects during the first and second exposures to each task was highly similar (with the exception of the go/no-go task, which produced a relatively low correlation). This indicated that the tasks were reliable, in themselves, in discriminating between the performances of individual animals. The preceding conclusion is supported by the finding that performance on each exposure to the contingency loaded onto the same factor with very similar loading weights. That performance on each task did not vary widely from first to second exposure implies that the tasks had a good test-retest reliability.

In discussing the validity of the tests, one can always claim that a set of reliable individual differences would have emerged if a different set of tasks had been employed. That is, the present tasks may not have been the best way to represent the dimension of response inhibition. However, it should be recognized that this argument could be made for any particular set of tasks, no matter how exhaustive. In addition, this argument could be run backwards, so that if any set of individual differences were established, it could be argued that they had arisen fortuitously-that the tasks chosen may have been the only ones from a wide range with the potential to demonstrate individual stable performance. More critically, it could be argued that the tasks employed shared common elements that produced differences in performance across groups of individuals, but that these differences were not along the dimension that they putatively measured. Such concerns make research in this area particularly difficult. However, if this potentially important question is to be addressed, a start must be made somewhere. The tasks employed in the present study span a wide range of procedures (classical and instrumental) and have themselves been used by others to investigate response inhibition (see Gray, 1987; Gray et al., 1979). Thus, they have, at least, face validity and criterion validity.

A number of accounts of human personality have been based, in part, on physiological interventions in nonhumans that reveal differences in performance between subjects possessing or lacking a particular brain region. It is supposed that humans share such systems with nonhumans, and that the efficacy of such systems in humans may well differ from individual to individual. Extrapolating from the present findings, it may be that results from experiments which demonstrate impairments in a number of tasks that appear to require the operation of a common system may not in fact be evidence that these tasks require the operation of a common system. There may be separate response inhibition systems for each task, since, in the absence of physiological intervention, rats' performances on these tasks appear to have very little in common. It should be acknowledged, however, that although the tasks used in the present study may be controlled primarily by independent systems, these systems could contribute to a common controlling system at some level which the present tasks were not measuring. Further research, including physiological interventions, will be required for this problem to be addressed.

It should be noted that the leap from the claim that a particular brain lesion disrupts performance to the claim that subjects may vary along a dimension imputed to be controlled by this brain region is fraught with potential difficulties. It may well be that the brain region does influence performance, but whether it does, or does not, control some aspect of the physical system required for that behavior, there is no need to assume a dimension of effectiveness in this system. In fact, studies of humans have similarly failed to find such dimensions based on physiological manipulations in nonhumans (e.g., Wilson et al., 1989). Of course, there could be all-or-none control over performance by the physiological system in question, with the gradations of performance reflecting the ability of other systems to influence performance on this task. However, this is not the manner in which such personality constructs are used in humans, since most measures of personality construct at least ordinal scales with which to measure the presence or absence of a trait (see, e.g., Eysenck \& Eysenck, 1985).

\section{REFERENCES}

CatTell, R. B. (1965). The scientific analysis of personality. London: Penguin.

Eysenck, H. J., \& Eysenck, M. W. (1985). Personality and individual differences. New York: Plenum.

GraY, J. A. (1987). The neuropsychology of emotion and personality. In 
S. D. Iversen, S. M. Stahl, \& E. C. Goodman (Eds.), Cognitive neurochemistry. Oxford: Oxford University Press.

GraY, J. A., Rawlins, J. N. P., \& FelDON, J. (1979). Brain mechanisms in the inhibition of behavior. In A. Dickinson \& R. A. Boakes (Eds.), Mechanisms of learning and motivation (pp. 295-316). Hillsdale, $\mathrm{NJ}$ : Erlbaum.

HunTINGFORD, F. A. (1976). The relationship between anti-predator behaviour and aggression among conspecifics in the three spined stickleback, Gasterosteus aculeatus. Animal Behaviour, 24, 245-260.

Mather, J. A., \& ANderson, R. C. (1993). Personalities of octopuses (Octopus rubescens). Journal of Comparative Psychology, 107, 336340.

Mischel, W. (1990). Personality dispositions revised and revisited: A review after three decades. In L. A. Pervin (Ed.), Handbook of personality: Theory and research (pp. 111-134). New York: Guilford Press.
Shoda, Y., Mischel, W., \& Wright, J. C. (1993). The role of situational demands and cognitive competencies in behavioral organization and personality coherence. Journal of Personality \& Social Psychology, 65, 1023-1035.

Wanchisen, B. A., Tatham, T. A., \& Mooney, S. E. (1989). Variable ratio conditioning history produces high- and low-rate fixed-interval performance in rats. Journal of the Experimental Analysis of Behavior, 52, 167-195.

WEST, R. (1991). Computing for psychologists: Statistical analysis using SPSS and MINITAB. London: Harwood

Wilson, G. D., BarretT, P. T., \& Gray, J. A. (1989). Human reactions to reward and punishment: A questionnaire examination of Gray's personality theory. British Joumal of Psychology, 80, 509-515.

(Manuscript received December 17, 1993; revision accepted for publication November 2, 1994.) 